\title{
On Estimation of HPD Interval for the Generalized Variance Using a Weighted Monte Carlo Method
}

\author{
Hea-Jung Kim ${ }^{1)}$
}

\begin{abstract}
Regarding to inference about a scalar measure of internal scatter of $p$-variate normal population, this paper considers an interval estimation of the generalized variance, $|\Sigma|$. Due to complicate sampling distribution, fully parametric frequentist approach for the interval estimation is not available and thus Bayesian method is pursued to calculate the highest probability density (HPD) interval for the generalized variance. It is seen that the marginal posterior distribution of the generalized variance is intractable, and hence a weighted Monte Carlo method, a variant of Chen and Shao (1999) method, is developed to calculate the HPD interval of the generalized variance. Necessary theories involved in the method and computation are provided. Finally, a simulation study is given to illustrate and examine the proposed method.
\end{abstract}

Keywords : HPD interval, generalized variance, weighted Monte Carlo method

\section{Introduction}

In applications the generalized variance of the $p$-variate normal population, $|\Sigma|$, is sometimes used as a scalar measure of internal scatter when $p \geq 2(|\Sigma|$ reduces to the ordinary variance when $p=1$ ). It also used to rank distinct groups or populations in order of their dispersion or spread. For example, a certain article, such as semiconductor, produced by a number of companies is characterized by a vector of $p$ measurements. Although the same product is produced on the average, the companies can be distinguished on the basis of their associated covariance matrices. Thus one can rank the supplying companies by ranking the normal populations using the generalized variance. The usage of the generalized variance has been widely accepted by statisticians (see Grizzle and Allen 1969, Press 1982 and Rencher 1995). However, due to complex sampling distribution involved in inferencing $|\Sigma|$, the analysis of $|\Sigma|$ is yet to be seen in applied settings.

This paper, therefore, presents a Bayesian approach to coming at a simple inference about

1) Professor, Department of Statistics, Dongguk University, Seoul 100-715.

E-mail : kim3hj@dongguk.edu. 
$|\Sigma|$. This is done by deriving the joint posterior distribution of the eigen values of $V^{-1} \Sigma$, where $V$ is the mean corrected sums of squares and cross product matrices obtained from a data set. Under the posterior distribution, we are driven to do multidimensional integration to evaluate marginal posterior summaries of $|\Sigma|$. Among them the present paper focuses on the evaluation of the HPD (highest probability density) interval of $|\Sigma|$. Noting that $|\Sigma|$ is a nonlinear function of the eigen values (inducing lack of invariance property), we can't expect analytical and exact numerical evaluation of the HPD interval (see Box and Tiao 1992). As an alternative solution, we develop a weighted Monte Carlo (WMC) method, a variant of Chen and Shao (1999) method, to compute the HPD interval of $|\Sigma|$. Finally, an illustrative example is given to demonstrate the accuracy of the proposed method.

\section{The Posterior Distribution}

Suppose that the random vector $X$ has a $p$-variate normal distribution, $N_{p}(\mu, \Sigma)$, and let $X_{1}, \ldots, X_{N}$ be a random sample of size $N$ on $X$. Then if

$$
\bar{X}=\frac{1}{N} \sum_{j=1}^{N} X_{j} \text { and } V=\sum_{j=1}^{N}\left(X_{j}-\bar{X}\right)\left(X_{j}-\bar{X}\right)^{\prime},
$$

the joint density of $\bar{X}$ and $V$ is given by

$$
p(\bar{X}, V \mid \mu, \Sigma) \propto \frac{|V|^{(N-p-2) / 2}}{|\Sigma|^{N / 2}} \operatorname{etr}\left\{-\frac{1}{2} \Sigma^{-1}\left[V+N(\bar{X}-\mu)(\bar{X}-\mu)^{\prime}\right]\right\}
$$

where $\operatorname{etr}\{A\}=\exp \{\operatorname{tr} A\}$. To assure very little information is actually contributed the analysis by a subjective prior density, we assume diffuse prior

$$
p(\mu, \Sigma) \propto|\Sigma|^{-(p+1) / 2} \text {. }
$$

Then the joint posterior density is

$$
p(\mu, \Sigma \mid \bar{X}, V) \propto|\Sigma|^{-(N+p+1) / 2} \operatorname{etr}\left\{-\frac{1}{2} \Sigma^{-1}\left[V+N(\bar{X}-\mu)(\bar{X}-\mu)^{\prime}\right]\right\} .
$$

Integrating (2.3) with respect to $\mu$, we have the marginal posterior distribution of $\Sigma$ :

$$
\Sigma \mid \bar{X}, V \sim W_{p}^{-1}(V, N+p) \text { for } N>p
$$

an inverted Wishart distribution with positive definite scale matrix $V$ and $N+p$ degrees of freedom (see, for instance, Raiffa and Schlaifer, 1961, p. 227).

Let the eigen values of $\Sigma$ are the components of $\lambda=\left(\lambda_{1}, \cdots, \lambda_{p}\right)^{\prime}$, $\lambda_{1}>\lambda_{2}>\cdots>\lambda_{p}>0$, and let the orthogonal decompositions of $\Sigma$ and $V$ are $H \Lambda H$ and $Q L Q^{\prime}$, respectively. Then the joint posterior density function of the latent roots $\lambda_{1}, \ldots, \lambda_{p}$ of $\Sigma$ can be derived by using Theorem 3.2.17 of Muirhead(1982):

$$
p(\lambda \mid \bar{X}, V)=c_{1} \prod_{i}^{p}\left(\frac{\ell_{i}}{\lambda_{i}}\right)^{(N+p) / 2} J(\lambda) \int_{\alpha(p)} \operatorname{etr}\left\{-\frac{1}{2} V H \Lambda^{-1} H^{\prime}\right\}(d H)
$$




$$
=c_{1} \prod_{i=1}^{p}\left(\frac{\ell_{i}}{\lambda_{i}}\right)^{(N+p) / 2} \exp \left\{-\frac{1}{2} \sum_{i=1}^{b}\left(\frac{\ell_{i}}{\lambda_{i}}\right)\right\} J^{*}(\lambda), \quad \lambda_{1}>\cdots>\lambda_{p}>0,
$$

where $c_{1}^{-1}=\pi^{-p^{2} / 2} 2^{n p / 2} \prod_{i=1}^{p} \ell_{i}{ }^{(p+1) / 2} \Gamma_{p}\{p / 2\} \Gamma_{p}\{n / 2\}$,

$\Lambda=\operatorname{diag}\left\{\lambda_{1}, \lambda_{2}, \cdots, \lambda_{p}\right\}, L=\operatorname{diag}\left\{l_{1}, l_{2}, \cdots, l_{p}\right\}, J(\lambda)=\prod_{i\langle j}^{0}\left(\lambda_{i}-\lambda_{j}\right)$, and

$J^{*}(\lambda)=J(\lambda) \int_{O(p)} \operatorname{etr}\left\{-\frac{1}{2}\left(L \tilde{H} \Lambda^{-1} \widehat{H}-\sum_{i=1}^{0} \ell_{i} / \lambda_{i}\right)\right\}(d \ddot{H})$ with $\vec{H}=Q^{\prime} H \in O(p)$,

a group of orthogonal $p \times p$ matrices, and $\Gamma_{p}(\cdot)$ is the multivariate gamma function (Press 1982).

The joint posterior density of $\lambda$ is quite complicated, and hence Bayesian inference about an arbitrary function of $|\Sigma|=\prod_{i=1}^{0} \lambda_{i}$ is a challenging problem. Specifically, the integral in (2.5) cannot be analytically evaluated, so that this prevents us using not only exact method, but a Monte Carlo method for the computation of posterior quantities of $|\Sigma|$. Thus we need an alternative and simple method for evaluating the quantities.

\section{Estimation of the HPD Interval}

The basic idea behind the alternative method is described as follows. Let

$R\left(\pi_{\alpha}\right)=\left\{|\Sigma|: P(|\Sigma| \mid \bar{X}, V) \geq \pi_{a}\right\}$ be the $(1-\alpha) \times 100 \%$ HPD interval of $|\Sigma|$, where $\pi_{\alpha}$ is the largest constant such that $\operatorname{Pr}\left(|\Sigma| \in R\left(\pi_{\alpha}\right)\right) \geq 1-\alpha$. Since exact marginal posterior distribution of $|\Sigma|$ is not known, we are not able to obtain $R\left(\pi_{a}\right)$. Therefore, instead of directly calculating the interval, we obtain the interval from the joint posterior density of $\delta=\left(\delta_{1}, \cdots, \delta_{p}\right)^{\prime}$, where $\delta_{1}>\delta_{2}>\cdots>\delta_{p}>0$ are the eigen values of $V^{-1} \Sigma$. For this, we derive exact joint posterior distribution of $\delta$ by choosing a "simultaneous diagonalization" transformation. Since $|\Sigma|=|V| \prod_{i=1}^{n} \delta_{i}$ and $R\left(\pi_{a}\right)$ is invariant with respect to a linear transformation of $|\Sigma|$, the HPD of $\prod_{i=1}^{n} \delta_{i}$ directly yields that of $|\Sigma|$.

\subsection{Posterior Density of Eigen Values of $V^{-1} \Sigma$}

The following lemma is useful for deriving the joint posterior density of eigen values of $V^{-1} \Sigma$.

Lemma 1. Let $\Sigma>0$ and $V>0$. Then there exist a matrix $C$, such that 


$$
C \Sigma C^{\prime}=D_{\delta}=\operatorname{diag}\left\{\delta_{1}, \cdots, \delta_{p}\right\}, C V C^{\prime}=I_{p}, \text { and }|\Sigma|=|V| \prod_{i=1}^{n} \delta_{i},
$$

where the $\delta_{i}{ }^{\prime}$ s are the eigen values of $|\Sigma-\delta V|=0$.

Proof. The proof is straightforward if we use the simultaneous diagonalization in Olkin and Tomsky (1981). In other words, let $\Gamma D_{\delta} \Gamma^{\prime}$ be the orthogonal decomposition of $V^{-1 / 2} \Sigma V^{-1 / 2}$ and let $C=\Gamma^{\prime} V^{-1 / 2}$, then the results hold.

Theorem 1. Let $X_{1}, \ldots, X_{N}$ be a random sample of size $N$ on $X \sim N_{p}(\mu, \Sigma)$. Then, under the prior (2.2), the joint posterior density function of the eigen values of $V^{-1} \Sigma$ is

$$
p(\delta \mid \bar{X}, V)=c_{0} \prod_{i}^{p} \delta_{i}^{-(N+p) / 2} \exp \left\{-\frac{1}{2} \sum_{i=1}^{b} \delta_{i}^{-1}\right\} J(\delta), \quad \delta_{1}>\cdots>\delta_{p}>0,
$$

where

$$
c_{0}^{-1}=\pi^{-p^{2} / 2} 2^{(N-1) p / 2} \Gamma_{p}\{(N-1) / 2\} \Gamma_{p}\{p / 2\}, \quad \delta=\left(\delta_{1}, \cdots, \delta_{p}\right)^{\prime}, \quad J(\delta)=\prod_{u<v}^{0}\left(\delta_{u}-\delta_{v}\right) .
$$

Proof. From Lemma 1, we see that , $D_{\delta}=C \Sigma C^{\prime}=\Gamma^{\prime} M \Gamma, M=V^{-1 / 2} \Sigma V^{-1 / 2}$ and $\Gamma$ is an orthogonal matrix so that $M=\Gamma D_{\delta} \Gamma^{\prime}$. Transformation of $\Sigma$ in (2.4) to $M$, we obtain the posterior distribution of $M, M \mid \bar{X}, V \sim W^{-1}\left(I_{p}, N+p\right)$ (see Press 1982, p.120, for the property of the inverted Wishart distribution). Transforming $M$ to $\delta$ and utilizing Theorem 3.2.17 of Muirhead (1982), we obtain the joint posterior density of $\delta_{1}, \cdots, \delta_{\triangleright}$ :

$$
p(\delta \mid \bar{X}, V)=c_{0} \prod_{i}^{p} \delta_{i}^{-(N+p) / 2} J(\delta) \int_{\alpha(p)} \operatorname{etr}\left\{-\frac{1}{2} \Gamma D_{\delta}^{-1} \Gamma^{\prime}\right\}(d \Gamma), \quad \delta_{1}>\cdots>\delta_{p}>0 .
$$

Since $\int_{\alpha(p)} \operatorname{etr}\left\{-\frac{1}{2} \Gamma D_{\delta}^{-1} \Gamma^{\prime}\right\}(d \Gamma)=\operatorname{etr}\left\{-\frac{1}{2} D_{\delta}^{-1}\right\} \int_{\alpha(p)}(d \Gamma)=\operatorname{etr}\left\{-\frac{1}{2} D_{\delta}^{-1}\right\}$ and the eigen values of $M=V^{-1 / 2} \Sigma V^{-1 / 2}$ is equivalent to those of $V^{-1} \Sigma$, we have the result. The distribution in (3.1) enables us to obtain various posterior quantities of the form

$$
\left.E\left[h\left(|V| \prod_{i}^{0} \delta_{i}\right) \mid \bar{X}, V\right)\right]=\int_{R^{p}} h\left(|V| \prod_{i=1}^{0} \delta_{i}\right) p(\delta \mid \bar{X}, V) d \delta
$$

where $h(\cdot)$ is a real valued function. The integral-type posterior quantities include posterior mean, posterior variance, higher order moments, and quantiles of $|\Sigma|=|V| \prod_{i=1}^{n} \delta_{i}$. The HPD interval can be also expressed as a function of the integral-type posterior quantity under certain conditions: A $(1-\alpha)$ HPD interval of $|\Sigma|=|V| \prod_{i=1}^{n} \delta_{i}$ is given by

$$
R\left(\pi_{a}\right)=\left\{|V| \xi: P(\xi \mid \bar{X}, V) \geq \pi_{a}\right\}
$$


where $\xi=\prod_{i=1}^{0} \delta_{i}$ and $\pi_{a}$ is the largest constant such that

$$
\int_{R^{\circ}} I\left\{|V| \xi \in R\left(\pi_{\alpha}\right)\right\} P(\delta \mid \bar{X}, V) d \delta \geq 1-\alpha
$$

When $P(\xi \mid \bar{X}, V)$ is continuous and unimodal, a $100(1-\alpha) \%$ HPD interval is $\left(\xi_{L}, \xi_{U}\right)$ where $\xi_{L}$ and $\xi_{U}$ are the solution to the following optimization problem $\min _{\xi_{L}<\xi_{U}}\left|P\left(\xi_{U} \mid \bar{X}, V\right)-P\left(\xi_{L} \mid \overline{X, V}\right)\right|+\left|F\left(\xi_{U} \mid \overline{X, V}\right)-F\left(\xi_{L} \mid \bar{X}, V\right)-(1-\alpha)\right|$.

\subsection{Weighted Monte Carlo Estimation of the HPD Interval}

As seen in (3.1) and (3.5), the analytic evaluation of $R\left(\pi_{\alpha}\right)$ is not available, because closed forms of the posterior pdf and df of $\xi$ are not known. Moreover, computing the HPD interval is difficult and challenging, because computation of $R\left(\pi_{\alpha}\right)$ requires knowing $\pi_{\alpha}$ and then calculating the content defined by (3.3). Recently, however, Chen and Shao (1999) propose a simple Monte Carlo (MC) method applicable for the computation of the HPD interval. The merit of Chen and Shao's method is that the method does not require a closed-form expression of the marginal posterior distribution of $\xi$. Instead we only need an importance function of the joint posterior distribution of $\delta$ to calculate the HPD interval.

Assume that $\left\{\delta^{(j)}, j=1, \cdots, m\right\}$ is an MCMC sample from an appropriate importance function $g(\delta)$. Then we can obtain an HPD interval for $\xi=\prod_{i=1}^{0} \delta_{i}$ as follows: Let $\left\{\xi_{j}, j=1, \cdots, m\right\}$ be a MCMC sample calculated from $\left\{\delta^{(j)}, j=1, \cdots, m\right\}$. Also let the $\xi_{(j)}$ denote the ordered values of the $\xi_{j}$. Then the $\gamma$ th quantile of the marginal posterior distribution of $\xi$ can be estimated by

$$
\hat{\xi}^{(\gamma)}=\left\{\begin{array}{lll}
\xi_{(1)} & \text { if } & \gamma=0 \\
\xi_{(j)} & \text { if } & \sum_{k=1}^{j-1} w_{(k)}<\gamma \leq \sum_{k=1}^{j} w_{(k)},
\end{array}\right.
$$

where $w_{(k)}$ is the weight function associated with the $k$ th ordered value $\xi_{(k)}$. More specifically, we first compute

$$
w_{k}=\frac{p\left(\delta^{(k)} \mid \bar{X}, V\right) / g\left(\delta^{(k)}\right)}{\sum_{\ell=1}^{m} p\left(\delta^{(\ell)} \mid \bar{X}, V\right) / g\left(\delta^{(\ell)}\right)} .
$$

Then we rewrite $\left\{w_{k}, k=1, \cdots, m\right\}$ as $\left\{w_{(k)}, k=1, \cdots, m\right\}$ so that the $k$ th value of $w_{(k)}$ corresponds to the $k$ th ordered value $\xi_{(k)}$. Using (3.6) we compute

$$
R_{k}(m)=\left(\xi^{(k / m)}, \hat{\xi}^{((k+[(1-\alpha) m] l)}\right)
$$


for $k=1,2, \cdots, m-[(1-\alpha) m]$ and a $100(1-\alpha) \%$ HPD interval of $\xi$ is $R_{k^{*}} \cdot(m)$ that has the smallest interval width among all $R_{k}(m)^{\prime}$ s. Here $[x]$ denotes the largest integer part of $x$. From (3.7) it is easy to observe that it is required to know the joint posterior density $p\left(\delta^{(\ell)} \mid \bar{X}, V\right)$ only up to a normalizing constant, since this normalizing constant cancels out in the calculation of $w_{k}$.

The most natural candidate for an important function $g(\delta)$ is the joint density of the order statistics from a random sample of inverse chi-squared variates. Apart from the $J(\delta)$ term, the density in (3.1) has the shape of the joint density of order statistics from $p$ independently and identically distributed inverse chi-squared variates with $N+p-2$ degrees of freedom, $\delta_{i} \stackrel{\text { iid }}{\underset{1}{-2}} \chi_{N+p-2}^{-2}, i=1, \ldots, p$. This similarity in shape is exploited in developing the importance sampling procedure.

Under the importance function the Gibbs sampler is consisting of a sequence of truncated inverse chi-squared distributions, so that, for $i=1, \ldots, p$, the full conditional distributions are

$$
\left[\delta_{i}^{(j)} \mid \delta_{1}^{(j)}, \ldots, \delta_{i-1}^{(j)}, \delta_{i+1}^{(j-1)}, \ldots, \delta_{p}^{(j-1)}\right] \sim \chi_{N+p-2}^{-2} \mathrm{I}\left(\delta_{i-1}^{(j)}<\delta_{i}^{(j)}<\delta_{i+1}^{(j-1)}\right)
$$

where $\delta^{(j)}=\left(\delta_{1}^{(j)}, \ldots, \delta_{p}^{(j)}\right)^{\prime}$. A simple accept/reject algorithm applies for generating the constrained inverse chi-squared variate $\delta_{i}^{(j)}$. Furthermore, the weight in (3.7) reduces to

$$
w_{k}=J\left(\delta^{(k)}\right) / \sum_{\ell=1}^{m} J\left(\delta^{(\ell)}\right),
$$

where $J\left(\delta^{(\ell)}\right)=\prod_{u<v}^{\natural}\left(\delta_{u}^{(\ell)}-\delta_{v}^{(\ell)}\right)$

\section{A Simulation Study}

In this section, we study the performance of the WMC estimator of the HPD interval for $|\Sigma|$ described in the forgoing section. We consider Bayesian inference concerning the generalized variance of two dependent normal populations. The reason for considering the bivariate normal population is that an exact confidence interval for $|\Sigma|$ is reasonably tractable by use of Fisher's $Z$ transformation. Suppose that $N$ independent observation vectors, $X_{i}$, $i=1, \ldots, N$, are drawn from the bivariate normal population $N_{2}(\mu, \Sigma)$, where $\mu=(.1,1)^{\prime}$ and $\Sigma=\left\{\sigma_{k \ell}\right\}$ with $\sigma_{11}=\sigma_{22}=1$ and $\sigma_{12}=\rho$. Since $|\Sigma|=1-\rho^{2}$, we can obtain an approximate confidence interval of $|\Sigma|$ by using a frequentist approach: Using Fisher's $Z$ transformation, we obtain $(1-\alpha) \times 100 \%$ approximate confidence interval for $|\Sigma|$ as 


$$
\left\{1-\left(\frac{e^{2 U_{\sigma}}-1}{e^{2 U_{a}}+1}\right)^{2}, 1-\left(\frac{e^{2 L_{a}}-1}{e^{2 L_{\alpha}}+1}\right)^{2}\right\}
$$

where $\quad U_{\alpha}=\frac{1}{2} \ln \left(\frac{1+r}{1-r}\right)+z_{\alpha / 2} / \sqrt{N-3}, \quad L_{\alpha}=\frac{1}{2} \ln \left(\frac{1+r}{1-r}\right)-z_{\alpha / 2} / \sqrt{N-3}, \quad$ and $\quad r$ denotes the sample correlation coefficient (see Press 1982). It is not difficult to compute the $(1-\alpha) \times 100 \%$ HPD interval for the generalized variance using the vague prior (2.2). Assume that $\left\{\delta^{(\ell)} ; \ell=1, \ldots, m\right\}, m=1000$, is a Gibbs sample from (3.9) with $p=2$ and 1000 burn-in Gibbs iterates. Many standard diagnostic measures (see Cowles and Carlin, 1996 and Kim, 1999) have been calculated to monitor the convergence of the Gibbs sampling algorithm and those indicated rapid convegence within $1000 \mathrm{Gibbs}$ iterates. The Gibbs sample yields the WMC estimator of the HPD interval for $|\Sigma|=1-\rho^{2}, \quad\left(|V| \xi^{\left(k^{*} / m\right)},|V| \xi^{\left(\left\{k^{*}+[(1-a) m]\right\}\right)}\right)$, where $\left(\xi^{\left(k^{*} / m\right)}, \xi^{\left(\left\{k^{*}+[(1-\alpha) m]\right\}\right)}\right)$ is that of the HPD interval for $\left|V^{-1} \Sigma\right|$.

Assuming that (4.1) conveges to the exact confidence interval of $|\Sigma|=1-\rho^{2}$ for large sample $(N=500,1000,5000)$ and the vague prior (2.2) is dominated by the large sample, we study convergence of the estimated HPD interval. To study the convergence of $\left(\theta_{L}^{*}, \theta_{U}^{*}\right)=\left(|V| \xi^{\left(k^{*} / m\right)},|V| \xi^{\left(\left\{k^{*}+[(1-\alpha) m]\right)\right)}\right)$, we define the following mean relative error (ME):

$$
M E_{N}=E\left[\left|\theta_{L}^{*}-\theta_{L}(\alpha)\right|+\left|\theta_{U}^{*}-\theta_{U}(\alpha)\right|\right] /\left(\theta_{U}(\alpha)-\theta_{L}(\alpha)\right)
$$

where $\left(\theta_{L}(\alpha), \theta_{U}(\alpha)\right)$ is the confidence interval (4.1) obtained from a sample of size $N=1000$ and the expectation is taken with respect to the posterior distribution of $\delta$. Since the expectation is analytically intractable, we use a standard simulation technique to estimate this expectation. We run $K$ simulations and then calculate

$$
M E_{N, k}=E\left[\left|\theta_{L, k}^{*}-\theta_{L}(\alpha)\right|+\left|\theta_{U, k}^{*}-\theta_{U}(\alpha)\right|\right] /\left(\theta_{U}(\alpha)-\theta_{L}(\alpha)\right)
$$

for $k=1, \ldots, K$. Then, $M E_{N}$ is approximated by $(1 / K) \sum_{k=1}^{K} M E_{N, k}$ and simulation standard error is the square root of the sample variance of the $M E_{N, k}{ }^{\prime}$ s. Table 1 gives the $M E_{N}^{\prime} \mathrm{s}$ with the simulation standard errors for various $N,|\Sigma|$ and $1-\alpha$ using $K=500$. From Table 1, it can be observed that the disagreement between the estimated HPD and exact confidence interval is within $10 \%$ of the length of the exact one for all cases. 
Table 1. Mean Relative Errors of the Estimated HPD intervals of $|\Sigma|$ with Simulation Standard Errors in Parentheses

\begin{tabular}{|c|c|c|c|c|c|}
\hline \multirow{2}{*}{$|\Sigma|$} & \multirow{2}{*}{$1-\alpha$} & \multirow{2}{*}{$\left(\theta_{L}(\alpha),\left(\theta_{U}(\alpha)\right)\right.$} & \multicolumn{3}{|c|}{$M E_{N}$} \\
\hline & & & $N=500$ & $N=1000$ & $N=5000$ \\
\hline \multirow[t]{3}{*}{.36} & .75 & $(.3443, .3867)$ & $\begin{array}{c}.0711 \\
(.0024)\end{array}$ & $\begin{array}{c}.0584 \\
(.0019)\end{array}$ & $\begin{array}{l}.0392 \\
(.0011)\end{array}$ \\
\hline & .90 & $(.3357, .3963)$ & $\begin{array}{r}.0598 \\
(.0021)\end{array}$ & $\begin{array}{c}.0447 \\
(.0015)\end{array}$ & $\begin{array}{l}.0343 \\
(.0009)\end{array}$ \\
\hline & .95 & $(.3302, .4024)$ & $\begin{array}{r}.0501 \\
(.0023)\end{array}$ & $\begin{array}{c}.0453 \\
(.0014)\end{array}$ & $\begin{array}{l}.0326 \\
(.0007)\end{array}$ \\
\hline \multirow[t]{3}{*}{.64} & .75 & $(.6115, .6674)$ & $\begin{array}{r}.0524 \\
(.0031)\end{array}$ & $\begin{array}{c}.0501 \\
(.0025)\end{array}$ & $\begin{array}{l}.0395 \\
(.0013)\end{array}$ \\
\hline & .90 & $(.5996, .6794)$ & $\begin{array}{r}.0558 \\
(.0026)\end{array}$ & $\begin{array}{c}.0496 \\
(.0022)\end{array}$ & $\begin{array}{l}.0389 \\
(.0011)\end{array}$ \\
\hline & .95 & $(.5920, .6871)$ & $\begin{array}{r}.4298 \\
(.0025)\end{array}$ & $\begin{array}{c}.0475 \\
(.0023)\end{array}$ & $\begin{array}{l}.0371 \\
(.0012)\end{array}$ \\
\hline \multirow[t]{3}{*}{.84} & .75 & $(.8182, .8668)$ & $\begin{array}{r}.0541 \\
(.0029)\end{array}$ & $\begin{array}{c}.0435 \\
(.0022)\end{array}$ & $\begin{array}{l}.0298 \\
(.0015)\end{array}$ \\
\hline & .90 & $(.8072, .8766)$ & $\begin{array}{r}.0473 \\
(.0024)\end{array}$ & $\begin{array}{c}.0426 \\
(.0025)\end{array}$ & $\begin{array}{l}.0285 \\
(.0016)\end{array}$ \\
\hline & .95 & $(.8009, .8826)$ & $\begin{array}{r}.0485 \\
(.0020)\end{array}$ & $\begin{array}{c}.0418 \\
(.0019)\end{array}$ & $\begin{array}{l}.0273 \\
(.0012)\end{array}$ \\
\hline \multirow[t]{3}{*}{.96} & .75 & $(.9432, .9716)$ & $\begin{array}{r}.0651 \\
(.0057)\end{array}$ & $\begin{array}{c}.0569 \\
(.0034)\end{array}$ & $\begin{array}{l}.0321 \\
(.0014)\end{array}$ \\
\hline & .90 & $(.9360, .9765)$ & $\begin{array}{r}.0501 \\
(.0038)\end{array}$ & $\begin{array}{c}.0473 \\
(.0025)\end{array}$ & $\begin{array}{l}.0305 \\
(.0010)\end{array}$ \\
\hline & .95 & $(.9312, .9794)$ & $\begin{array}{r}.0504 \\
(.0036)\end{array}$ & $\begin{array}{c}.0484 \\
(.0023) \\
\end{array}$ & $\begin{array}{l}.0303 \\
(.0011) \\
\end{array}$ \\
\hline
\end{tabular}

\section{Concluding Remarks}

In this paper we propose a Bayesian method for estimating the HPD interval for $|\Sigma|$, a scalar measure of internal scatter in $p$-variate normal population. Other competing measures proposed for the same purpose include $\operatorname{tr}\left(\Sigma^{2}\right)^{1 / 2}$ and the difference between the largest and smallest eigen values of $\Sigma$ (see Press 1982). Unfortunately, methodology from previous sections does not apply to calculate the HPD intervals for the other measures, because each of them is not a function of product of the eigen values of $V^{-1} \Sigma$. Thus we may need to develop other methods for calculating the HPD intervals for them.

However, the methodology proposed in this paper can easily extended to calculate HPD 
On Estimation of HPD Interval for the Generalized Variance Using a Weighted Monte Carlo Method 313

interval of a ratio of two generalized variances, $\left|\Sigma_{1}\right| /\left|\Sigma_{2}\right|$, a linear function of $\left|V_{1}^{-1} \Sigma_{1}\right| /\left|V_{2}^{-1} \Sigma_{2}\right|$, where $V_{1}$ and $V_{2}$ are the mean corrected sums of squares and cross product matrices obtained from respective samples of two independent multivariate normal populations with covariance matrices $\Sigma_{1}$ and $\Sigma_{2}$. The ratio is a useful criterion for comparing the dispersion or spread between two multivariate normal populations, and the extension pertaining to calculate the HPD interval of the ratio is now under investigation.

\section{Acknowledgement}

This work is supported by a research fund of Dongguk University. The author wishes to thank the editor and two anonymous referees for providing helpful comments.

\section{References}

[1] Box, G. E. P. and Tiao, G. C. (1992). Bayesian Inference in Statistical Analysis. New York: Wiley.

[2] Chen, M.-H, and Shao, Q.-M. (1999). Monte Carlo estimation of Bayesian credible and HPD intervals. Journal of the Computational and Graphical Statistics 8, 69-92.

[3] Cowles, M. K. and Carlin, B. P. (1996). Markov chain Monte Carlo convergence diagnostics: a comparative review, Journal of the American Statistical Association 91, 883-904.

[4] Grizzle, J. E. and Allen, D. M. (1969). Analysis of growth and does response curves. Biometrics 25, 357-382.

[5] Kim, H. J. (1999). A Bayesian variable selection method for binary response probit regression, Journal of the Korean Statistical Society 28, 167-182.

[6] Muirhead, R. J. (1982). Aspects of Multivariate Statistical Theory. New York: Wiely.

[7] Olkin, I. and Tomsky, J. L. (1981). A new class of multivariate tests based on the union-intersection principle. Annals of Statistics 9, 792-802.

[8] Press, S. J. (1982). Applied Multivariate Analysis. Florida: Krieger Publishing Co.

[9] Raiffa, H. and Schlaifer, R. (1961). Applied Statistical Decision Theory. Boston: Harvard University Press.

[10] Rencher, A. C. (1995). Methods of Multivariate Analysis. New York: Wiley. 